\title{
Correction to: Completely positive dynamical semigroups and quantum resonance theory
}

\author{
Martin Könenberg ${ }^{1,2} \cdot$ Marco Merkli $^{1}$
}

Published online: 24 April 2019

(c) Springer Nature B.V. 2019

\section{Correction to: Lett Math Phys (2017) 107:1215-1233 https://doi.org/10.1007/s11005-017-0937-z}

Correction. The bound (2.23) in Theorem 2.1 has to be replaced by

$$
\left|R_{\lambda, t}(X)\right| \leq C\left(|\lambda|+\lambda^{2} t\right) \mathrm{e}^{-\lambda^{2}(1+O(\lambda)) \gamma t}\|X\|
$$

Implication. The difference is that in reality we can only show $\lambda^{2} t$ on the right side, instead of the $|\lambda|^{3} t$ as announced in the published paper. The remainder (0.1) is still asymptotically exact (vanishing as $t \rightarrow \infty$ ), and our result still proves that the dynamics is approximated, asymptotically exactly, by a CPT semigroup. But for times $t \approx 1 / \lambda^{2}$, the approximation is not guaranteed to be small.

Nevertheless, as will be discussed elsewhere in more detail, we can obtain a proof of Theorem 2.1, exactly as stated in the published paper, if instead of allowing all observables $X \in \mathcal{B}\left(\mathcal{H}_{\mathrm{S}}\right)$, we restrict to $X$ which commute with $H_{\mathrm{S}}$. Such observables determine the dynamics of the system populations (diagonal elements of the reduced density matrix). This means that the true population dynamics is approximated by a CPT semigroup dynamics, uniformly in time to accuracy $O(\lambda)$, and the approximating Markovian dynamics is also asymptotically exact.

The original article can be found online at https://doi.org/10.1007/s11005-017-0937-z.

凶 Martin Könenberg

martin.koenenberg@mathematik.uni-stuttgart.de

Marco Merkli

merkli@mun.ca

1 Department of Mathematics and Statistics, Memorial University, St. John's, NL, Canada

2 Present Address: Fachbereich Mathematik, Universität Stuttgart, Stuttgart, Germany 
Proof of the correction (0.1). An estimate for the remainder term in the proof of Theorem 2.1, as given in the paper, contains a mistake. Namely, in footnote 5 [after Eq. (3.44)], the real phase of the exponential was omitted. The correct estimate is

$$
\left|\mathrm{e}^{\mathrm{i} t\left(e+\lambda^{2} a_{e, j}\right)}-\mathrm{e}^{\mathrm{i} t\left(\widetilde{e}+\lambda^{2} \widetilde{\lambda} \widetilde{e}, j\right.}\right| \leq C \lambda^{2} t \mathrm{e}^{-\lambda^{2}(1+O(\lambda)) \gamma t} .
$$

The corrected bound (0.2) yields (0.1) by the argument in the paper. We get $(0.2)$ as follows,

$$
\begin{aligned}
\left|\mathrm{e}^{\mathrm{i} t\left(e+\lambda^{2} a_{e, j}\right)}-\mathrm{e}^{\mathrm{i} t\left(\widetilde{e}+\lambda^{2} \widetilde{\lambda}_{\widetilde{e}, j}\right)}\right| & =\mathrm{e}^{-t \lambda^{2} \operatorname{Im} a_{e, j}}\left|1-\mathrm{e}^{\mathrm{i} t\left(\widetilde{e}-e+\lambda^{2}\left(\widetilde{\lambda}_{\widetilde{e}, j}-a_{e, j}\right)\right)}\right| \\
& =\mathrm{e}^{-t \lambda^{2} \operatorname{Im} a_{e, j}}\left|\int_{0}^{t\left(\widetilde{e}-e+\lambda^{2}\left(\widetilde{\lambda}_{\tilde{e}, j}-a_{e, j}\right)\right)} \mathrm{e}^{\mathrm{i} z} \mathrm{~d} z\right| \\
& \leq \mathrm{e}^{-t \lambda^{2} \operatorname{Im} a_{e, j}} t\left|\widetilde{e}-e+\lambda^{2}\left(\widetilde{\lambda}_{\widetilde{e}, j}-a_{e, j}\right)\right| \mathrm{e}^{t \lambda^{2}\left|\operatorname{Im}\left(\widetilde{\lambda}_{\widetilde{e}, j}-a_{e, j}\right)\right|} .
\end{aligned}
$$

Using in the last inequality that $\operatorname{Im} a_{e, j}=(1+O(\lambda)) \gamma$, that $\widetilde{\lambda}_{\widetilde{e}, j}-a_{e, j}=O(\lambda)$ and that

$$
\widetilde{e}-e=O\left(\lambda^{2}\right)
$$

gives (0.2). We note that by Lemma 3.1 in the published paper, the bound (0.3) above is seemingly only $O(\lambda)$. However, since $\rho_{\mathrm{S}, \beta, \lambda}-\rho_{\mathrm{S}, \beta, 0}=O\left(\lambda^{2}\right)$ (the linear term vanishes since the interaction has vanishing average in the reservoir vacuum state), we have $\widetilde{H}_{\mathrm{S}}-H_{\mathrm{S}}=O\left(\lambda^{2}\right)$. The $O(\lambda)$ bounds in Lemma 3.1 are thus actually $O\left(\lambda^{2}\right)$ bounds and (0.3) holds.

Publisher's Note Springer Nature remains neutral with regard to jurisdictional claims in published maps and institutional affiliations. 